\title{
Motesanib Diphosphate in Progressive Differentiated Thyroid Cancer
}

\author{
Steven I. Sherman, M.D., Lori J. Wirth, M.D., Jean-Pierre Droz, M.D., \\ Michael Hofmann, M.D., Ph.D., Lars Bastholt, M.D., Renato G. Martins, M.D., \\ Lisa Licitra, M.D., Michael J. Eschenberg, M.S., Yu-Nien Sun, Ph.D., \\ Todd Juan, Ph.D., Daniel E. Stepan, M.D., and Martin J. Schlumberger, M.D., \\ for the Motesanib Thyroid Cancer Study Group*
}

A BSTRACT

\section{BACKGROUND}

The expression of vascular endothelial growth factor (VEGF) is characteristic of differentiated thyroid cancer and is associated with aggressive tumor behavior and a poor clinical outcome. Motesanib diphosphate (AMG 706) is a novel oral inhibitor of VEGF receptors, platelet-derived growth-factor receptor, and KIT.

\section{METHODS}

In an open-label, single-group, phase 2 study, we treated 93 patients who had progressive, locally advanced or metastatic, radioiodine-resistant differentiated thyroid cancer with $125 \mathrm{mg}$ of motesanib diphosphate, administered orally once daily. The primary end point was an objective response as assessed by an independent radiographic review. Additional end points included the duration of the response, progression-free survival, safety, and changes in serum thyroglobulin concentration.

\section{RESULTS}

Of the 93 patients, 57 (61\%) had papillary thyroid carcinoma. The objective response rate was $14 \%$. Stable disease was achieved in $67 \%$ of the patients, and stable disease was maintained for 24 weeks or longer in $35 \%$; $8 \%$ had progressive disease as the best response. The Kaplan-Meier estimate of the median duration of the response was 32 weeks (the lower limit of the $95 \%$ confidence interval [CI] was 24; the upper limit could not be estimated because of an insufficient number of events); the estimate of median progression-free survival was 40 weeks ( $95 \%$ CI, 32 to 50 ). Among the 75 patients in whom thyroglobulin analysis was performed, $81 \%$ had decreased serum thyroglobulin concentrations during treatment, as compared with baseline levels. The most common treatment-related adverse events were diarrhea (in $59 \%$ of the patients), hypertension (56\%), fatigue (46\%), and weight loss $(40 \%)$.

CONCLUSIONS

Motesanib diphosphate can induce partial responses in patients with advanced or metastatic differentiated thyroid cancer that is progressive. (ClinicalTrials.gov number, NCT00121628.)
From the University of Texas M.D. Anderson Cancer Center, Houston (S.I.S.); Dana-Farber Cancer Institute, Boston (L.J.W.); Centre Léon Bérard, Lyon, France (J.-P.D.); Medical School Bern, Bern, Switzerland (M.H.); Odense University Hospital, Odense, Denmark (L.B.); University of Washington, Seattle (R.G.M.); Istituto Nazionale dei Tumori, Milan (L.L.); Amgen, Thousand Oaks, CA (M.J.E., Y.-N.S., T.J., D.E.S.); and Institut Gustave Roussy, University Paris Sud, Villejuif, France (M.J.S.). Address reprint requests to $\mathrm{Dr}$. Sherman at the Department of Endocrine Neoplasia and Hormonal Disorders, University of Texas M.D. Anderson Cancer Center, Unit 435, 1515 Holcombe Blvd., P.O. Box 301402, Houston, TX 77230-1402, or at sisherma@mdanderson.org.

*Additional investigators who participated in this study are listed in the Appendix.

N Engl J Med 2008;359:31-42. Coppright $\odot 2008$ Massachusetts Medical Society. 
T HYROID CANCERS ARE CLASSIFIED HIStologically into four groups: papillary, follicular, medullary, and undifferentiated or anaplastic thyroid carcinomas. ${ }^{1}$ Papillary and follicular carcinomas (including the Hürthle-cell variant) are collectively known as differentiated thyroid cancers, ${ }^{1,2}$ and they account for approximately $95 \%$ of incident cases. ${ }^{3}$ Initial treatment typically involves total thyroidectomy followed by long-term administration of levothyroxine at doses that suppress thyrotropin; adjuvant radioiodine $\left.{ }^{(131} \mathrm{I}\right)$ is often administered as well. ${ }^{2-4}$ This regimen is usually effective, but the 10 -year recurrence rate is 20 to $30 \%$ among patients who are older, among patients with tumors larger than $4 \mathrm{~cm}$ in diameter, and among patients in whom the tumor extends beyond the thyroid or in whom extensive lymph-node metastases develop. ${ }^{4}$ There is no effective treatment for radioiodine-resistant metastatic disease; the 10-year survival rate in these cases is less than $15 \% .^{5}$

Increased expression of vascular endothelial growth factor (VEGF), a potent angiogenesis stimulator, is characteristic of differentiated thyroid cancers ${ }^{6,7}$ and is associated with increased growth, progression, and invasiveness of the tumor and with decreased recurrence-free survival. ${ }^{4,8-10}$ For this reason, an inhibitor of angiogenesis may be effective when initial therapy fails. ${ }^{11}$ Motesanib diphosphate (Amgen) is an oral inhibitor of the tyrosine kinases of VEGF receptors 1, 2, and 3; platelet-derived growth-factor receptor; and KIT. ${ }^{12}$ In a phase 1 study, treatment with $125 \mathrm{mg}$ of motesanib diphosphate once daily resulted in antitumor activity in patients with advanced solid cancers, including five patients with differentiated thyroid cancer. ${ }^{13}$ In the present study, we investigated the efficacy and tolerability of motesanib diphosphate in progressive, locally advanced or metastatic differentiated thyroid cancer.

\section{METHODS}

\section{PATIENTS}

We included in the study adults who had histologically confirmed, locally advanced or metastatic differentiated thyroid cancer and documented evidence of disease progression (based on two sets of radiographic images) according to the Response Evaluation Criteria in Solid Tumors (RECIST) ${ }^{14}$ as assessed by the investigator, with- in 6 months before entry into the study. A rising serum thyroglobulin level alone was not sufficient evidence of disease progression before enrollment. Other key inclusion criteria were the presence of at least one lesion that was defined as measurable according to the RECIST guidelines and that was not amenable to surgical resection or external-beam radiation therapy or was refractory to radioiodine; the absence of untreated brain metastases; an Eastern Cooperative Oncology Group performance status score of 0 to 2; and adequate hepatic, renal, and cardiac function. Patients were ineligible if they had ever received treatment with VEGF-receptor inhibitors or if they had received nonhormonal anticancer therapy within 30 days before the start of the study. The protocol was approved by each center's independent ethics committee or institutional review board, and all patients provided written informed consent before enrollment.

\section{STUDY DESIGN AND END POINTS}

This phase 2, open-label study involving a cohort of patients with differentiated thyroid cancer was conducted at 42 centers in 10 countries. A parallel cohort included similar patients with metastatic medullary thyroid cancer; data from this cohort were not included in the analysis reported here. The primary end point was an objective response according to RECIST, as assessed by a centralized, independent review. Additional end points included the duration of response, progressionfree survival, the time to response, and overall survival; adverse events; pharmacokinetic characteristics; and changes in the serum thyroglobulin concentration.

Patients received $125 \mathrm{mg}$ of motesanib diphosphate orally once daily for up to 48 weeks or until there was evidence of unacceptable toxicity or disease progression. Treatment was offered beyond 48 weeks in an extension study if a clinical benefit was observed. Therapy was withheld if a treatment-related grade 3 adverse event that could not be controlled with supportive care, any related grade 4 adverse event, or symptomatic hypertension occurred. Treatment could be resumed at a dose of $100 \mathrm{mg}$ per day, if toxicity was reduced to a grade of 1 or less for nonhematologic toxicity or to a grade of 2 or less for hematologic toxicity, or at a dose of $75 \mathrm{mg}$ per day after a second interruption of treatment that was related to toxicity. 
Adverse events were classified according to the Medical Dictionary for Regulatory Activities, and severity was graded according to the National Cancer Institute Common Terminology Criteria for Adverse Events, version 3.0. Patients had regular assessments of blood pressure (weekly until week 6 , then every other week until week 16 , and every 4 weeks thereafter), blood chemical profiles, blood counts, and urine protein levels. Free thyroxine and thyrotropin levels were measured at baseline and monthly thereafter.

\section{ASSESSMENT OF TUMOR RESPONSE}

Computed tomographic or magnetic resonance imaging scans of the neck, chest, and abdomen were obtained at 8-week intervals and when progression of the disease was suspected. An objective response (complete or partial response according to RECIST) was assessed by a centralized, independent review, with confirmation by repeat imaging studies at least 4 weeks later. A bone scintigram that showed no new osseous metastases (as compared with the findings at study entry) was also required to define an objective response in patients with follicular or Hürthle-cell carcinoma. The designation of stable disease required a single assessment of either stable disease or an unconfirmed objective response 47 days or more after the first administration of the drug.

\section{PHARMACOKINETICS}

Plasma samples to assess pharmacokinetics were collected from nine patients 15 minutes, $30 \mathrm{~min}$ utes, and 1, 2, 4, 6, 8, and 24 hours after the administration of the drug on day 1 of the study. To assess trough concentrations of motesanib after long-term administration, plasma was collected before the daily dose at 4-week intervals during the first 24 weeks of the study. Plasma motesanib concentrations were measured by means of liquid chromatography coupled with tandem mass spectroscopy (CEDRA Clinical Research).

\section{THYROGLOBULIN ANALYSES AND TUMOR GENOTYPING}

Blood samples were collected at baseline and at 4-week intervals, and serum thyroglobulin measurements were performed with the use of the Access 2 automated immunochemiluminometric assay (Beckman Coulter). Intraassay coefficients of variation were $3.3 \%, 1.7 \%$, and $1.9 \%$ at 0.76 , 7.0, and 106 ng per milliliter, respectively; inter- assay coefficients of variation over the 9-month period of measurement were $4.8 \%, 4.7 \%$, and $5.0 \%$ at $0.75,7.3$, and $106 \mathrm{ng}$ per milliliter, respectively.

Tumor DNA from 33 patients was centrally screened for mutations in RET, B-type Raf kinase (BRAF), HRAS, KRAS, NRAS, and phosphatidylinositol-3-kinase, catalytic, alpha polypeptide (PIK3CA), and for rearrangements of RET-PTC1, RET-PTC3, and PAX8-peroxisome proliferator-activated receptor gamma 1 (PPAR 1 1). A detailed description of the methods is included in the Supplementary Appendix (available with the full text of this article at www. nejm.org).

\section{STATISTICAL ANALYSIS}

We planned to enroll at least 80 patients to achieve a two-sided $95 \%$ confidence interval of 12 to $30 \%$ for the rate of an objective response, assuming an observed rate of $20 \%$. All patients who received one dose or more of motesanib diphosphate were included in the efficacy and safety analyses. The overall tumor response was derived from multiple time-point responses as assessed by an independent radiographic review. The duration of the tumor response was the interval between the first, subsequently confirmed, objective response and evidence of progressive disease. The time to the response was the time from the first administration of the study drug to the initially documented (and subsequently confirmed) response. Progression-free survival was the time from the first administration of the drug to the date of radiographic evidence of disease progression or death. The duration of the response, progression-free survival, and overall survival were described with Kaplan-Meier estimates and $95 \%$ confidence intervals. The thyroglobulin analysis subgroup included all patients for whom baseline and one or more post-baseline measurements of thyroglobulin were available and who did not have detectable antithyroglobulin antibodies.

Amgen designed the study, in collaboration with the study cochairs, Drs. Sherman and Schlumberger. The academic authors had complete access to the study data and decided, with Amgen's support, to publish the study. Employees of Amgen collected and managed the data and performed the statistical analysis. An independent panel of radiologists from RadPharm, a commercial imaging laboratory, reviewed all radiographic assessments. Amgen and the study 
Table 1. Demographic and Baseline Characteristics of the Patients.

\section{Characteristic}

Sex - no. (\%)

Female

Male

Age - yr

Median

Range

Race or ethnic group - no. (\%)*

White

Asian

Hispanic or Latino

Black

Histologic subtype of differentiated thyroid cancer - no. (\%)

$$
\text { Papillary† }
$$

Hürthle cell

Follicular

Others

Time from initial diagnosis - yr $\S$

Median

Range

Extent of disease - no. (\%)

Locally advanced

Metastatic

No. of sites of disease - no. (\%)

1

2

$\geq 3$

ECOG performance status - no. (\%) 9

0

1

2

Prior therapy for thyroid cancer - no. (\%)

Thyroidectomy\|

Radioiodine therapy

0 Courses

1 Course

2 Courses

$\geq 3$ Courses

External-beam radiation therapy

0 Unique sites

1 Unique site

$\geq 2$ Unique sites
All Patients ( $\mathrm{N}=93)$

44 (47)

49 (53)

62

36-81

85 (91)

$4(4)$

3 (3)

1 (1)

17 (18)

15 (16)

$4(4)$

4.4

$0.4-21.3$

1 (1)

92 (99)

26 (28)

39 (42)

$28(30)$

47 (51)

37 (40)

9 (10)

93 (100)

3 (3)

14 (15)

35 (38)

41 (44)

40 (43)

32 (34)

21 (23) 


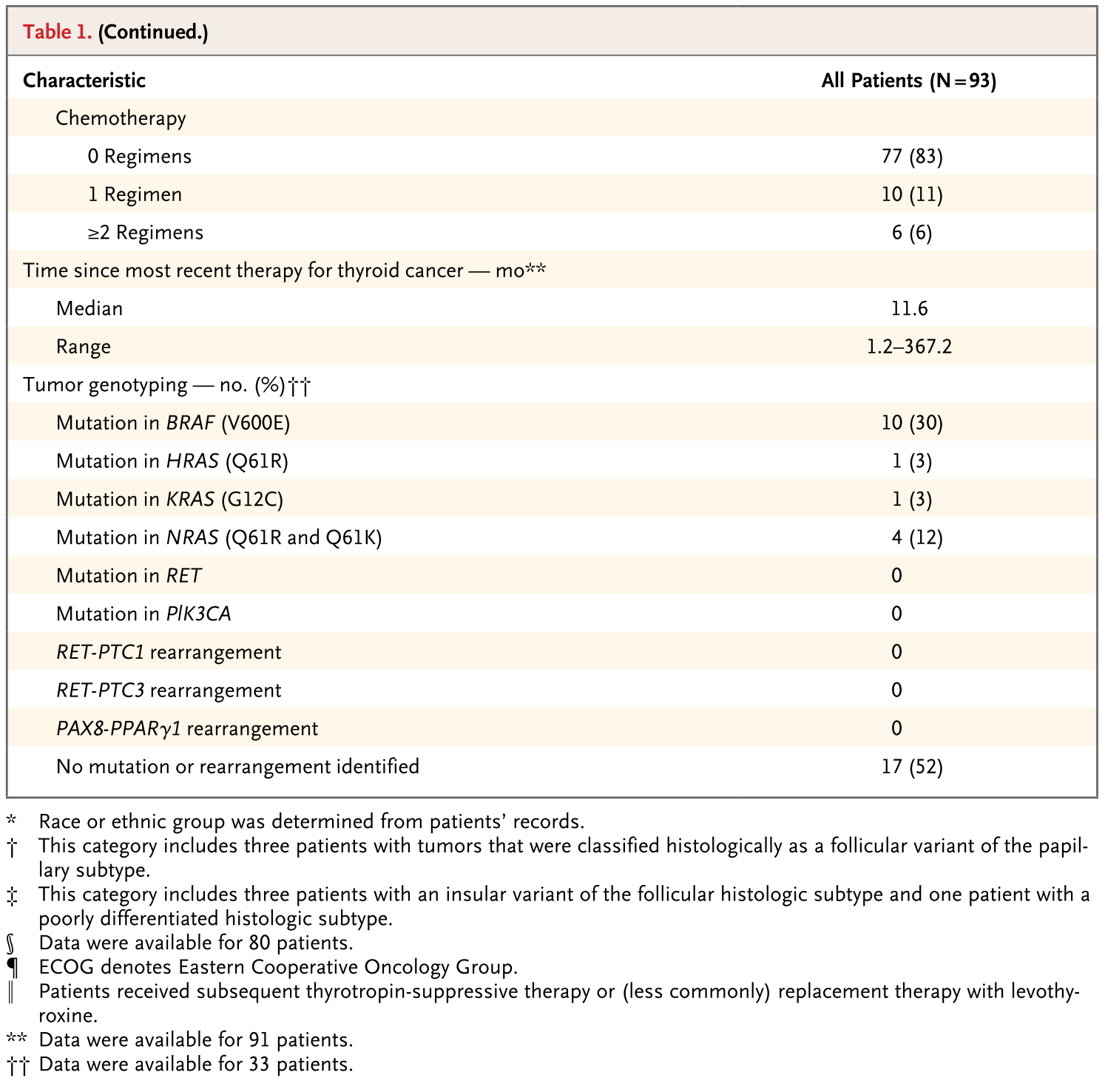

cochairs interpreted the data. The manuscript was written by the study cochairs, with assistance from Complete Healthcare Communications and Amgen and with contributions from all of the coauthors. The study cochairs attest to the completeness and accuracy of the data.

\section{RESULTS}

\section{PATIENTS}

Between July 29, 2005, and March 13, 2006, a total of 93 patients with differentiated thyroid cancer were enrolled in the study. All of the patients received one dose or more of motesanib diphosphate. A total of 32 patients completed 48 weeks of treatment. The remaining patients discontinued the drug because of disease progres- sion (35 patients), adverse events (12), death (5), withdrawal of consent (1), an administrative decision (1), a protocol deviation (1), or the patient's request (6). The median length of treatment for all patients was 35 weeks (range, 0.4 to 56), and the median follow-up was 50 weeks (range, 1 to 77).

The most common histologic subtype was papillary thyroid carcinoma. At study entry, almost all of the patients had metastases, usually with multiple sites of disease, including lung $(87 \%)$, lymph nodes $(72 \%)$, and liver (26\%). Disease progression according to RECIST within 6 months before study day 1 was documented in all patients by the investigator. Seventeen percent of the patients had previously received systemic chemotherapy (Table 1).

Table 1 summarizes the results of tumor geno- 
typing, which are shown in detail in Table 3 in the Supplementary Appendix. Of 25 patients with papillary thyroid carcinoma, 10 had tumors carrying the mutant BRAF V600E oncogene, and 4 had mutations in RAS genes (HRAS and KRAS in 1 patient each and NRAS in 2 patients).

\section{EFFICACY}

The primary end point of a confirmed objective response was achieved in 13 patients (14\%; $95 \%$ confidence interval [CI], 7.7 to 22.7), all of whom had partial responses; the median time to the response was 15 weeks (95\% CI, 8 to 27) (Table 2). Stable disease was observed in 62 patients (67\%), with durable stable disease (i.e., disease that was stable for 24 weeks or more) in 33 patients (35\%). Nine patients $(10 \%)$ had unconfirmed partial responses that were classified as stable disease. Overall, $49 \%$ of the patients had either a confirmed partial response or durable stable disease. Disease progression was the best observed response in seven patients (8\%). In 69 patients (74\%), tumor measurements decreased from the baseline measurements (Fig. 1).

Of the 13 patients with an objective response, 4 had disease that subsequently progressed while the patients were taking the study medication. The Kaplan-Meier estimate of the median duration of a response was 32 weeks (the lower limit of the $95 \%$ CI was 24; the upper limit could not be estimated because of an insufficient number of events). The estimated median progression-free survival was 40 weeks ( $95 \%$ CI, 32 to 50) (Fig. 2A). The median overall survival was not estimable; the estimate of the survival rate at 12 months was $73 \%$ (95\% CI, 63 to 83) (Fig. 2B).

There was no significant difference in the rate of the objective response between patients with the papillary or follicular variant of papillary carcinoma (57 patients) and those with the follicular or Hürthle-cell type (36 patients) (12\% and $17 \%$, respectively; $\mathrm{P}=0.56$ ). We found no association between the presence or absence of the BRAF V600E mutation (10 and 15 patients, respectively) and the clinical outcome. Six of the 10 patients whose tumor contained the BRAF mutation had either a confirmed partial response or durable stable disease, as compared with 5 of the 15 patients without the mutation (Table 3 in the Supplementary Appendix).

\section{SAFETY}

A total of 87 patients $(94 \%)$ had at least one treatment-related adverse event during the course of the study (Table 3). Twelve patients (13\%) discontinued treatment owing to adverse events. The most commonly reported events were diarrhea, hypertension, fatigue, and weight loss; patients with hypertension were treated with antihypertensive medication, and those with diarrhea, fatigue, or weight loss received supportive care. Grade 3 events were reported in 51 patients (55\%); 5 patients had a total of eight treatment-related grade 4 events (hypocalcemia in 2 patients and hyperuricemia, hypokalemia, cerebral hemorrhage, confusion, agitation, and oliguria in 1 patient each). There were two treatment-related deaths; both were due to pulmonary hemorrhage and occurred in patients in whom the disease had progressed. Several treatment-related events of interest (previously observed with anti-VEGF or anti-VEGF receptor therapies) were noted (Table 3). Increased thyrotropin concentrations, hypothyroidism, or both were reported in $22 \%$ of the patients. Five patients (5\%) had cholecystitis (grade 2 in three patients and grade 3 in two patients). Two patients had treatment-related cardiac disorders; one had bradycardia, palpitations, and tachycardia, and one had myocardial ischemia (grade 2 in both patients).

\section{PHARMACOKINETICS}

Motesanib was rapidly absorbed, with a median time to the maximal plasma concentration $\left(\mathrm{C}_{\max }\right)$ of 1.0 hour; the terminal half-life was approximately 6.7 hours. The mean ( \pm SD) motesanib $C_{\max }$ value $(787 \pm 526 \mathrm{ng}$ per milliliter $[2.10 \pm 1.41 \mu \mathrm{mol}$ per liter]) and area under the plasma concentration-time curve $\left(\mathrm{AUC}_{0-24}, 3.99 \pm 1.42 \mu \mathrm{g} \times\right.$ hour per milliliter $[10.7 \pm 3.79 \mu \mathrm{mol} \times$ hour per liter]) were similar to those observed for the $125-\mathrm{mg}$ dose in another study of motesanib as monotherapy for solid tumors. ${ }^{13}$ Between weeks 4 and 24, the median trough plasma concentration $\left(\mathrm{C}_{\mathrm{min}}\right)$, measured in 9 to 23 patients at different time points, ranged from 8.50 to 26.3 ng per milliliter (22.7 to $70.3 \mathrm{nmol}$ per liter) and was consistently above the $\mathrm{IC}_{50}$ value (4 ng per milliliter $[10 \mathrm{nmol}$ per liter]) for inhibition of the proliferation of human umbilical-vein endothelial cells in vitro. $^{12}$ 
Table 2. Tumor Response According to RECIST and Independent Review.*

\begin{tabular}{|c|c|}
\hline Variable & All Patients $(\mathrm{N}=93)$ \\
\hline \multicolumn{2}{|l|}{ Assessment of response - no. (\%) } \\
\hline Complete response & 0 \\
\hline Partial response & $13(14)$ \\
\hline Stable disease & $62(67)$ \\
\hline Unconfirmed partial response classified as stable disease $\dagger$ & $9(10)$ \\
\hline Durable stable disease ( $\geq 24 \mathrm{wk}$ ) & $33(35)$ \\
\hline Progressive disease & $7(8)$ \\
\hline No data on response availableł & $11(12)$ \\
\hline Objective response - \% $(95 \% \mathrm{Cl})$ & $14(7.7-22.7)$ \\
\hline Clinical benefit - no. (\%)』 & $46(49)$ \\
\hline \multicolumn{2}{|l|}{ Time to tumor response - wk } \\
\hline Median & 15 \\
\hline $95 \% \mathrm{Cl}$ & $8-27$ \\
\hline \multicolumn{2}{|l|}{ Duration of partial response } \\
\hline Censored data - no./total no. (\%) & $8 / 13(62)$ \\
\hline Patients who had a relapse - no./total no. (\%) & $5 / 13(38)$ \\
\hline Kaplan-Meier estimate of median duration - wk $(95 \% \mathrm{Cl})$ & $32(24-N E)$ \\
\hline \multicolumn{2}{|l|}{ Progression-free survival } \\
\hline Alive with no progression — no. (\%) & $43(46)$ \\
\hline Progression or death - no. (\%) & $50(54)$ \\
\hline Kaplan-Meier estimate of median progression-free survival — wk $(95 \% \mathrm{Cl})$ & $40(32-50)$ \\
\hline \multicolumn{2}{|l|}{ Kaplan-Meier estimate of progression-free survival rate — \% $(95 \% \mathrm{Cl})$} \\
\hline Week 16 & $78(69-87)$ \\
\hline Week 32 & $60(50-71)$ \\
\hline Week 48 & $37(24-50)$ \\
\hline \multicolumn{2}{|l|}{ Overall survival } \\
\hline Patients who survived — no. (\%) & $66(71)$ \\
\hline Patients who died — no. (\%) & $27(29)$ \\
\hline Kaplan-Meier estimate of median overall survival - mo $(95 \% \mathrm{Cl})$ & NE \\
\hline \multicolumn{2}{|l|}{ Kaplan-Meier estimate of survival rate - \% $(95 \% \mathrm{Cl})$} \\
\hline Month 4 & $89(82-95)$ \\
\hline Month 8 & $82(74-90)$ \\
\hline Month 12 & $73(63-83)$ \\
\hline
\end{tabular}

*NE denotes not estimable (insufficient number of events).

$\uparrow$ Seven patients had a single assessment of partial response with a subsequent assessment of progressive disease or had no additional tumor assessment; two patients with follicular or Hürthle-cell carcinoma did not have the required bone scans to confirm partial response.

$\downarrow$ No week 8 scans were available (scans were obtained before day 47 or no radiographic assessments were performed), available scans were not interpretable, or no baseline scans were available.

$\int$ Clinical benefit was defined as either a confirmed partial response or durable stable disease.

q Data for patients with a confirmed objective response and no assessment of disease progression during the study are included as censored data at the time of the last disease assessment. 


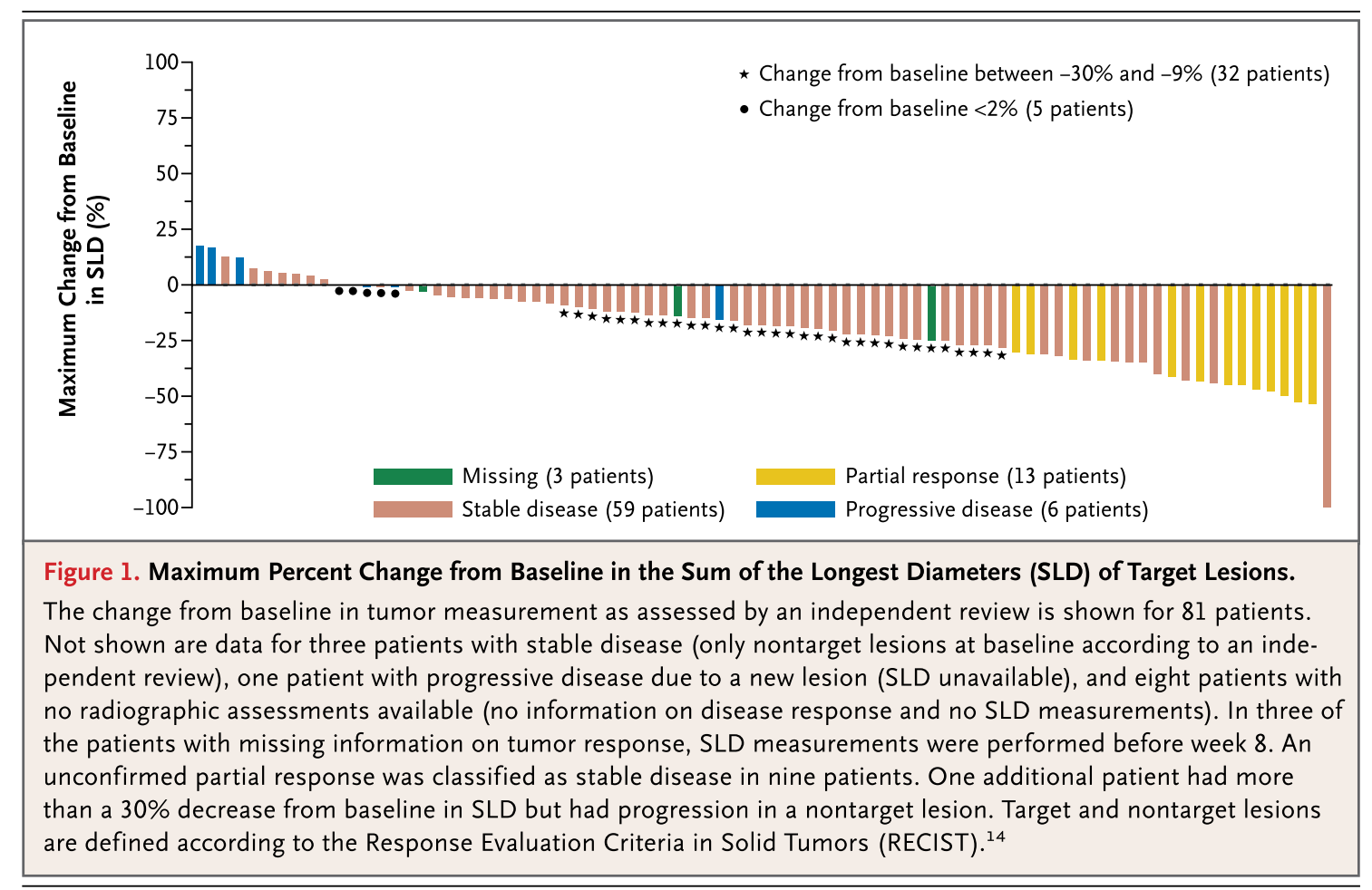

\section{THYROGLOBULIN ANALYSES}

Of the 75 patients in whom thyroglobulin analysis was performed, 61 (81\%) had serum thyroglobulin concentrations that decreased from baseline during the study. In 34 patients (45\%) the decrease was $50 \%$ or more, and that decrease was sustained for 24 weeks or more in 11 patients (15\%). A correlation was observed between a decrease from baseline of $50 \%$ or more in thyroglobulin concentration and a decrease from baseline of $30 \%$ or more in the sum of the longest diameter of target tumor lesions (Spearman's rank correlation coefficient, $0.472 ; \mathrm{P}<0.001$ ).

\section{DISCUSSION}

The treatment for progressive, advanced or metastatic differentiated thyroid cancer has been limited to surgery, radioiodine therapy, external-beam radiation therapy, or a combination of these treatments. In patients with radioiodine-resistant disease, cytotoxic chemotherapy yields low response rates of short duration, is often associated with considerable toxicity, and does not prolong survival. ${ }^{15-17}$ Recently, angiogenesis has been explored as a target for the treatment of advanced thyroid cancer. A study of thalidomide, which has several actions, including the inhibition of angiogenesis, showed that 5 of 28 patients with a va- riety of thyroid cancers had unconfirmed partial responses on the basis of criteria other than RECIST. ${ }^{18}$

We found that 13 of 93 patients with progressive, radioiodine-resistant, locally advanced or metastatic differentiated thyroid cancer who received $125 \mathrm{mg}$ of motesanib diphosphate once daily had a partial response. Of these 93 patients, $87 \mathrm{had}$ at least one adverse event, and 51 had a grade 3 adverse event. The response to motesanib diphosphate, which inhibits multiple signaling pathways that are involved in differentiated thyroid tumorigenesis, including VEGF and plateletderived growth-factor receptors, ${ }^{12}$ suggests that inhibiting angiogenesis in differentiated thyroid cancer may be clinically useful. Recent preliminary data suggest that other inhibitors of VEGF receptors have efficacy in advanced thyroid cancer. ${ }^{19,20}$ In a single-institution trial of sorafenib, the confirmed partial response rate among 58 patients with metastatic papillary thyroid carcinoma was $3 \% .{ }^{20}$ In our study of 93 patients with progressive differentiated thyroid cancer, the proportion of patients who had stable disease (67\%), the proportion who had durable stable disease (35\%) and the median progression-free survival (40 weeks [95\% CI, 32 to 50]) all suggest clinically meaningful tumor control. In contrast, no partial or complete responses were seen in a 


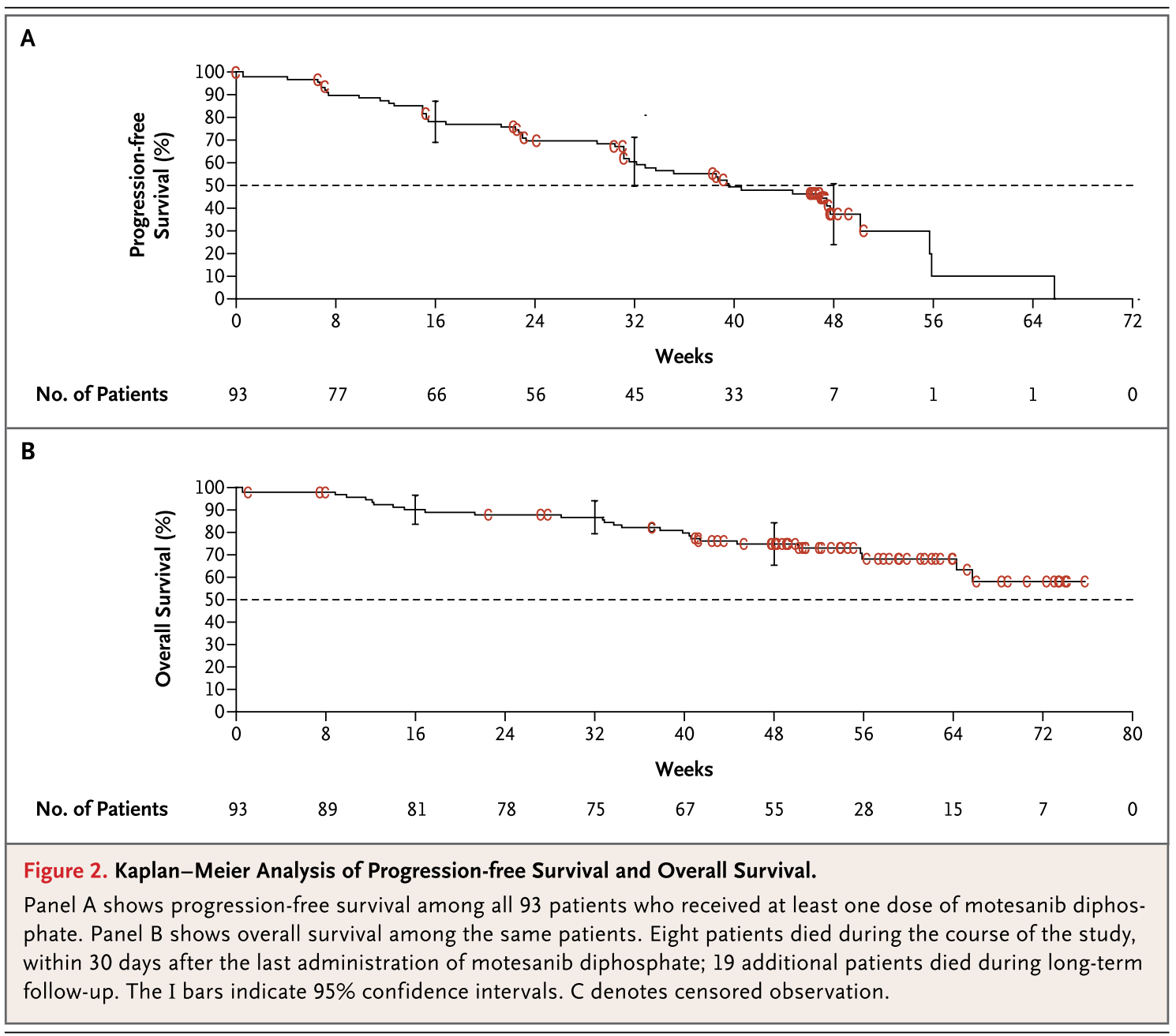

phase 2 study of gefitinib, an inhibitor of the epidermal growth factor receptor, among 18 patients with advanced differentiated thyroid cancer, and the progression-free survival time was only 3.7 months ( $95 \%$ CI, 1.8 to 5.7 ). ${ }^{21}$

Changes in the thyroglobulin concentration are used to monitor residual tumor volume, recurrent or metastatic tumor growth, or both in patients with differentiated thyroid cancer. ${ }^{22,23}$ The decrease in serum thyroglobulin levels that we found in most patients and its correlation with the tumor response are consistent with the findings previously reported for radioiodine therapy. ${ }^{24}$ The magnitude of this effect was probably underestimated because thyrotropin promotes thyroglobulin secretion, and treatment with motesanib diphosphate caused increased serum concentrations of thyrotropin.

We attempted to correlate the tumor genotype and clinical response in differentiated thyroid cancer, but the number of tumors available to be stud- ied was too small to draw conclusions about an association of responsiveness with BRAF mutations that influence the VEGF-signaling pathway. ${ }^{25}$

Two adverse events related to motesanib diphosphate treatment are worth mentioning. Hypothyroidism, an increase in the thyrotropin concentration, or both occurred in $22 \%$ of the patients and have recently been reported during treatment with the multikinase inhibitors sunitinib, ${ }^{26}$ imatinib, ${ }^{27}$ and possibly sorafenib. ${ }^{28}$ In athyreotic patients (patients whose thyroids were surgically resected), motesanib diphosphate may induce alterations in the absorption or metabolism of thyroxine instead of affecting thyroid hormone synthesis, a mechanism that is implicated in sunitinib-induced hypothyroidism. ${ }^{26}$ Given these multiple effects on thyroid function, we suggest close monitoring of thyrotropin levels and adjustments in the dose of levothyroxine, as appropriate, in patients who are receiving multikinase inhibitors. In five patients in our study $(5 \%)$, chole- 


\begin{tabular}{|c|c|c|c|c|}
\hline \multirow[t]{2}{*}{ Event } & All Grades & Grade 3 & Grade 4 & Grade 5 \\
\hline & \multicolumn{4}{|c|}{ no. of patients (\%) } \\
\hline \multicolumn{5}{|l|}{ All treatment-related adverse events } \\
\hline Adverse events & $87(94)$ & $51(55)$ & $5(5)$ & $2(2)$ \\
\hline Serious adverse events & $19(20)$ & $8(9)$ & $5(5)$ & $2(2)$ \\
\hline \multicolumn{5}{|l|}{$\begin{array}{l}\text { Treatment-related adverse events in } \geq 10 \% \text { of pa- } \\
\text { tients }\end{array}$} \\
\hline Diarrhea & $55(59)$ & $12(13)$ & 0 & 0 \\
\hline Hypertension & $52(56)$ & $23(25)$ & 0 & 0 \\
\hline Fatigue & $43(46)$ & $4(4)$ & 0 & 0 \\
\hline Weight loss & $37(40)$ & $5(5)$ & 0 & 0 \\
\hline Abdominal pain & $28(30)$ & $5(5)$ & 0 & 0 \\
\hline Nausea & $26(28)$ & $2(2)$ & 0 & 0 \\
\hline Anorexia & $25(27)$ & $4(4)$ & 0 & 0 \\
\hline Headache & $24(26)$ & $3(3)$ & 0 & 0 \\
\hline Dry mouth & $13(14)$ & 0 & 0 & 0 \\
\hline Hypothyroidism & $11(12)$ & 0 & 0 & 0 \\
\hline Vomiting & $11(12)$ & $1(1)$ & 0 & 0 \\
\hline Increased thyrotropin & $11(12)$ & 0 & 0 & 0 \\
\hline Asthenia & $10(11)$ & 0 & 0 & 0 \\
\hline Arthralgia & $9(10)$ & $1(1)$ & 0 & 0 \\
\hline \multicolumn{5}{|l|}{ Related adverse events of interest** } \\
\hline $\begin{array}{l}\text { Hypothyroidism, increased thyrotropin, } \\
\text { or both }\end{array}$ & $20(22)$ & 0 & 0 & 0 \\
\hline Hemorrhage & $13(14)$ & $1(1)$ & $1(1)$ & $2(2)$ \\
\hline Gallbladder toxicity & $12(13)$ & $2(2)$ & 0 & 0 \\
\hline Thromboembolic events & $2(2)$ & $1(1)$ & 0 & 0 \\
\hline Cardiac disorders & $2(2)$ & 0 & 0 & 0 \\
\hline
\end{tabular}

* Adverse events of interest are those previously observed with anti-VEGF or anti-VEGF receptor therapies.

$\dagger$ Gallbladder toxicity included biliary cholic, cholecystitis, acute cholecystitis, cholelithiasis, gallbladder enlargement, and gallbladder edema.

$†$ Cardiac disorders included bradycardia, palpitations, and tachycardia (in one patient) and myocardial ischemia (in one).

cystitis developed, a complication that has not previously been reported in association with antiangiogenic therapy. These five patients presented with classic symptoms (right-upper-quadrant pain and fever) and had a response to medical or surgical treatment (or both), including cholecystectomy. This incidence of cholecystitis is higher than expected on the basis of other studies of motesanib diphosphate; however, the cause is unknown and the relation to motesanib diphosphate is under investigation.

The key limitation of this trial was the lack of randomization with a control group. Because the study group specifically included patients with advanced differentiated thyroid cancer, traditional comparator therapies, such as cytotoxic chemotherapy, were inappropriate owing to their ineffectiveness and toxicity. However, we believe that the large number of patients enrolled in this study allowed a reasonable estimate of clinical benefit.

In conclusion, motesanib diphosphate may be an effective treatment in some patients with progressive, metastatic, radioiodine-resistant differentiated thyroid cancer. However, a broader applicability of treatment that inhibits angiogenesis 
in thyroid cancer needs to be established in further studies.

\section{A DDEN DUM}

Therapy with the multikinase inhibitor sorafenib has been reported to yield a $26 \%$ partial response rate in patients with metastatic differentiated thyroid carcinoma. ${ }^{29}$

Supported by Amgen.

Presented in part at the annual meeting of the American Society of Clinical Oncology, Chicago, June 1 to 5, 2007; and at the annual meeting of the European Thyroid Association, Leipzig, Germany, September 1 to 5, 2007.

Dr. Sherman reports receiving consulting fees from Abbott, AstraZeneca, Bayer, Bristol-Myers Squibb, Eisai, Enzon, Exelixis, and Genzyme, honoraria and lecture fees from Abbott and Genzyme, and grant support from Amgen, Genzyme, and AstraZeneca, and serving on an advisory committee for AstraZeneca and Abbott; Dr. Wirth, lecture fees from Sanofi-Aventis; Dr. Droz, lecture fees from AstraZeneca and consulting and lecture fees from Sanofi-Aventis; Dr. Bastholt, consulting and lecture fees from AstraZeneca, Pfizer, and Schering-Plough; Dr. Martins, honoraria and lecture fees from Genentech and Eli Lilly and grant support from Amgen, Genentech, Eli Lilly, Infinity, Pfizer, ImClone, Dana-Farber, Novartis, and Exelixis, and serving on advisory committees or review panels for Genentech; Dr. Licitra, consulting fees from GlaxoSmithKline, consulting and lecture fees from Merck, and grant support and consulting and lecture fees from Associazione Italiana Ricerca Cancro (AIRC); Dr. Schlumberger, consulting and lecture fees from AstraZeneca, Exelixis, and Genzyme and grant support from AstraZeneca, Genzyme, and Amgen; and Mr. Eschenberg and Drs. Sun, Juan, and Stepan being employees of and having equity ownership and stock options in Amgen. No other potential conflict of interest relevant to this article was reported.

We thank Carole Spencer, Ph.D., for measurement of thyroglobulin and analysis and discussion of the results; Jennifer Britton and Monica MacDonald for study management; Ali Hassan, Ph.D., whose work was funded by Amgen, and Beate D. Quednau, Ph.D. (Amgen), for assistance in drafting the manuscript; and David Reese, M.D., for discussion of the study results and critical review of the manuscript.

\section{APPENDIX}

In addition to the authors, the following investigators participated in the study: University Hospital for Nuclear Medicine and Endocrinology, Klagenfurt, Austria - P. Lind; University Hospital for Nuclear Medicine and Endocrinology, Salzburg, Austria - C. Pirich; University Hospital St. Luc, Brussels - C. Daumerie; Institut Gustave Roussy, Villejuif, France — E. Baudin; Institut Bergonié, Bordeaux, France - B.N. Bui; Centre Hospitalier Universitaire (CHU) de la Timone, Marseille, France - B. Conte-Devolx; CHU Angers, Angers, France - V. Rohmer; Institut Jean Godinot, Reims, France - C. Schvartz; Belgyogyaszati Hospital, Budapest, Hungary - I. Szabolcs, K. Racz; University Hospital, Florence, Italy - M.L. Brandi; University Hospital, Pisa, Italy - A. Pinchera, R. Elisei; Hospital S. Luigi Gonzaga, Orbassano, Italy - F. Orlandi; University Hospital, Siena, Italy — F. Pacini; Maria Skłodowska-Curie Memorial Cancer Center and Institute of Oncology, Gliwice, Poland - B. Jarzab; Oddzial Clinic of Endocrinology, Poznan, Poland - J. Sowinski; Sahlgrenska University Hospital, Göteborg, Sweden - S. Jansson; Karolinska University Hospital, Stockholm — G. Lundell, A. Hallqvist; University Hospital, Geneva - C. Meier, J. Philippe; University of Pittsburgh Cancer Institute, Pittsburgh - S. Agarwala; Henry Ford Health System, Detroit - H. Ali; Mission International Medical Group, Mission Viejo, CA - J. Barrera; Center for Cancer and Blood Disorders, Bethesda, $M D$ - R. Boccia; Cleveland Clinic Foundation, Cleveland - R. Bukowski; Washington Hospital Center, Washington, DC - K. Burman; University ofCalifornia, San Francisco, San Francisco - O. Clark; Dartmouth-Hitchcock Medical Center, Lebanon, NH - T. Davis; University of Texas M.D. Anderson Cancer Center, Houston - A. Hoff, N. Sarlis; Lakeland Regional Cancer Center, Lakeland, FL - J. Jakub; Providence St. Joseph Medical Center, Burbank, CA - R. Mena; University of Cincinnati Barrett Cancer Center, Cincinnati — Z. Nahleh; Premiere Oncology Medical Corporation, Santa Monica, CA - L. Rosen; Cancer Center of the Carolinas, Greenville, SC - J. Stephenson; Sparrow Regional Cancer Center, Lansing, MI - G. Srkalovic; Pacific Shores Medical Group, Long Beach, $C A-\mathrm{N}$. Tchekmedyian.

REFERENCES

1. DeLellis RA. Pathology and genetics of thyroid carcinoma. J Surg Oncol 2006; 94:662-9.

2. Schlumberger MJ. Papillary and follicular thyroid carcinoma. N Engl J Med 1998;338:297-306.

3. Hundahl SA, Fleming ID, Fremgen AM, Menck HR. A National Cancer Data Base report on 53,856 cases of thyroid carcinoma treated in the U.S., 1985-1995. Cancer 1998;83:2638-48.

4. Cooper DS, Doherty GM, Haugen BR, et al. Management guidelines for patients with thyroid nodules and differentiated thyroid cancer. Thyroid 2006;16:109-42. 5. Durante C, Haddy N, Baudin E, et al. Long-term outcome of 444 patients with distant metastases from papillary and follicular thyroid carcinoma: benefits and limits of radioiodine therapy. J Clin Endocrinol Metab 2006;91:2892-9.

6. Bunone G, Vigneri P, Mariani L, et al. Expression of angiogenesis stimulators and inhibitors in human thyroid tumors and correlation with clinical pathological features. Am J Pathol 1999;155:1967-76.
7. Klein M, Picard E, Vignaud JM, et al. Vascular endothelial growth factor gene and protein: strong expression in thyroiditis and thyroid carcinoma. J Endocrinol 1999;161:41-9.

8. Kilicarslan AB, Ogus M, Arici C, Pestereli HE, Cakir M, Karpuzoglu G. Clinical importance of vascular endothelial growth factor (VEGF) for papillary thyroid carcinomas. APMIS 2003;111:439-43.

9. Lennard CM, Patel A, Wilson J, et al. Intensity of vascular endothelial growth factor expression is associated with increased risk of recurrence and decreased disease-free survival in papillary thyroid cancer. Surgery 2001;129:552-8.

10. Vieira JM, Santos SC, Espadinha C, et al. Expression of vascular endothelial growth factor (VEGF) and its receptors in thyroid carcinomas of follicular origin: a potential autocrine loop. Eur J Endocrinol 2005;153:701-9.

11. Fagin JA. How thyroid tumors start and why it matters: kinase mutants as targets for solid cancer pharmacotherapy. J Endocrinol 2004;183:249-56.
12. Polverino A, Coxon A, Starnes C, et al. AMG 706, an oral, multikinase inhibitor that selectively targets vascular endothelial growth factor, platelet-derived growth factor, and Kit receptors, potently inhibits angiogenesis and induces regression in tumor xenografts. Cancer Res 2006;66: 8715-21.

13. Rosen LS, Kurzrock R, Mulay M, et al. Safety, pharmacokinetics, and efficacy of AMG 706, an oral multikinase inhibitor, in patients with advanced solid tumors. J Clin Oncol 2007;25:2369-76.

14. Therasse P, Arbuck SG, Eisenhauer EA, et al. New guidelines to evaluate the response to treatment in solid tumors: European Organization for Research and Treatment of Cancer, National Cancer Institute of the United States, National Cancer Institute of Canada. J Natl Cancer Inst 2000;92:205-16.

15. Haugen BR. Management of the patient with progressive radioiodine nonresponsive disease. Semin Surg Oncol 1999; 16:34-41.

16. Gottlieb JA, Hill CS Jr. Chemotherapy 
of thyroid cancer with adriamycin: experience with 30 patients. N Engl J Med 1974; 290:193-7.

17. Droz JP, Schlumberger M, Rougier $P$, Ghosn M, Gardet P, Parmentier C. Chemotherapy in metastatic nonanaplastic thyroid cancer: experience at the Institut Gustave-Roussy. Tumori 1990;76:480-3. 18. Ain KB, Lee C, Williams KD. Phase II trial of thalidomide for therapy of radioiodine-unresponsive and rapidly progressive thyroid carcinomas. Thyroid 2007;17: 663-70.

19. Cohen EE, Rosen LS, Vokes EE, et al Axitinib is an active treatment for all histologic subtypes of advanced thyroid cancer: results from a phase II study. J Clin Oncol (in press)

20. Kloos R, Ringel M, Knopp $M$, et al. Significant clinical and biologic activity of RAF/VEGF-R kinase inhibitor BAY 43-
9006 in patients with metastatic papillary thyroid carcinoma (PTC): updated results of a phase II study. J Clin Oncol 2006;24: Suppl:288s. abstract.

21. Pennell NA, Daniels GH, Haddad RI, et al. A phase II study of gefitinib in patients with advanced thyroid cancer. Thyroid 2008;18:317-23.

22. Sherman SI. Thyroid carcinoma. Lancet 2003;361:501-11.

23. Spencer CA, LoPresti JS, Fatemi S, Nicoloff JT. Detection of residual and recurrent differentiated thyroid carcinoma by serum thyroglobulin measurement. Thyroid 1999;9:435-41

24. Sisson JC, Giordano TJ, Jamadar DA, et al. 131-I treatment of micronodular pulmonary metastases from papillary thyroid carcinoma. Cancer 1996;78:2184-92. 25. Jo YS, Li S, Song JH, et al. Influence of the BRAF V600E mutation on expression of vascular endothelial growth factor in papillary thyroid cancer. J Clin Endocrinol Metab 2006;91:3667-70.

26. Desai J, Yassa L, Marqusee E, et al. Hypothyroidism after sunitinib treatment for patients with gastrointestinal stromal tumors. Ann Intern Med 2006;145:660-4. 27. de Groot JW, Zonnenberg BA, van Ufford-Mannesse PQ, et al. A phase II trial of imatinib therapy for metastatic medullary thyroid carcinoma. J Clin Endocrinol Metab 2007;92:3466-9.

28. Robinson SI, Hobday TJ, Sathananthan A, Morris JC III, McWilliams RR. Can sorafenib cause hypothyroidism? J Chemother 2007;19:352-3.

29. Gupta-Abramson V, Troxel AB, Nellore $\mathrm{A}$, et al. Phase II trial of sorafenib in advanced thyroid cancer. J Clin Oncol (in press).

Copyright $\odot 2008$ Massachusetts Medical Society. 